\title{
Prospective Application of Nanoparticles Green Synthesized Using Medicinal Plant Extracts as Novel Nanomedicines
}

\section{Rajendran K Selvakesavan (D) Gregory Franklin (D)}

Institute of Plant Genetics of the Polish Academy of Sciences, Poznan, Poland
Correspondence: Gregory Franklin Institute of Plant Genetics of the Polish Academy of Sciences, 34 Strzeszynska street, Poznan, 60-479, Poland

$\mathrm{Tel}+48616550266$

Email fgre@igr.poznan.pl

\begin{abstract}
The use of medicinal plants in green synthesis of metal nanoparticles is increasing day by day. A simple search for the keywords "green synthesis" and "nanoparticles" yields more than 33,000 articles in Scopus. As of August 10, 2021, more than 4000 articles have been published in 2021 alone. Besides demonstrating the ease and environmental-friendly route of synthesizing nanomaterials, many studies report the superior pharmacological properties of green synthesized nanoparticles compared to those synthesized by other methods. This is probably due to the fact that bioactive molecules are entrapped on the surface of these nanoparticles. On the other hand, recent studies have confirmed the nanodimension and biocompatibility of metal ash (Bhasma) preparations, which are commonly macerated with biological products and administered for the treatment of various diseases in Indian medicine since ancient times. This perspective article argues for the prospective medical application of green nanoparticles in the light of Bhasma.
\end{abstract}

Keywords: green synthesis, nanoparticle, Bhasma, medicinal plants, phytonanomedicine

\section{Introduction}

Miniaturization is the central dogma of nanoscience, and the materials that are nanoscale in at least one dimension are nanomaterials. ${ }^{1}$ Surface area, size-to-charge ratio, reactivity, activation by visible light, thermal stability, conductivity, $\mathrm{pH}$, magnetic behavior, charge storage, etc. are greatly enhanced at the nanoscale of materials. $^{2}$ These physicochemical properties make nanomaterials more useful than their bulk forms in many fields such as industry, agriculture, engineering and medicine. $^{3,4}$

Nanoparticles open up new possibilities in modern medicine with many research advances. $^{5-7}$ In particular, their nano size helps the particles to easily reach the human cell, which promotes their application in disease detection to drug delivery. ${ }^{3,8,9}$ Organic nanoparticles synthesized from proteins, nucleic acids, lipids, carbohydrates and polymers are non-toxic and biocompatible. ${ }^{10}$ Although inorganic nanoparticles such as silver $(\mathrm{Ag})$, gold $(\mathrm{Au})$, copper $(\mathrm{Cu})$, zinc oxide $(\mathrm{ZnO})$, etc. are also used in medicine, their stability and biocompatibility need to be improved by coating or encapsulating them with some organic materials. ${ }^{10,11}$ Ferumoxytol is an example of iron (Fe) metal nanoparticles used in the treatment of anemia. ${ }^{12,13}$ The optical property of Au nanoparticles has been used in diagnostics. For example, the color change of Au nanoparticles due to aggregation by alkaline phosphatase has 
been used in the development of a colorimetric method to detect the concentration of ochratoxin $\mathrm{A},{ }^{14}$ indicating the expansion of the alkaline phosphatase-related disease detection technique. Organo-metallic nanoparticles are known for controlled and targeted delivery of drugs for the treatment of a number of diseases including cancer. $^{15-17}$

The use of nanomaterials in medicine dates back to ancient times. Ayurveda, a traditional system of medicine practiced in the Indian subcontinent since the $7^{\text {th }}$ century uses metal ash (Bhasma) to treat various diseases. ${ }^{18}$ Bhasma are metallic/ mineral preparations treated with herbal juices or decoctions and exposed to a certain amount of heat, as in the puta system of Ayurveda. Bhasmas are widely recommended in India for the treatment of many disease conditions. ${ }^{19}$ Bhasma preparation is a top-down approach often begins with the burning of metals, ores, minerals, etc. at very high temperature and repeated titration with plant extract or other organic materials for purification. ${ }^{20}$ Wet grinding of purified metal ash with plant extracts or powders in Bhasma preparation could create microscopic thermal cavities that allow secondary plant metabolites to be activated and act as chelating agents. Since Bhasma processing requires repeated cycles of high temperatures, eg, $600{ }^{\circ} \mathrm{C}$ in the final stages of preparation, ${ }^{19}$ secondary metabolites bound to the metal ash could be lost as a result of maceration. However, in Ayurvedic treatment practice, Bhasma is administered either alone or in combination with medicinal plant extracts or powders as required. ${ }^{21}$ Recent studies have shown that many of the Bhasma preparations are submicron nanoparticles and organometallic in nature. ${ }^{22,23}$ The biocompatibility of Bhasma has been demonstrated in recent studies. ${ }^{24,25}$ The metals showed toxic effects when present in macro size and showed medicinally beneficial effects when present in micro or nano size. ${ }^{26}$ The need for repeated washing, drying and puta steps makes the process of Bhasma preparation time consuming. ${ }^{19,22,25}$

Recent advances in green chemistry have developed simple, rapid and inexpensive methods for the synthesis of nanoparticles, popularly known as green synthesis. ${ }^{4}$ Green synthesis of nanoparticles can be performed both in vivo and in vitro using biomolecules (nucleic acids and proteins), ${ }^{27,28}$ microorganisms ${ }^{7,29}$ and plant extracts. ${ }^{30-32}$ In particular, extracts of various medicinal plants have been widely used for the reduction of metal ions to nanoparticles because of their phytochemical richness. ${ }^{33,34}$ The resulting nanoparticles are often found to be biocompatible and more bioactive than their counterparts synthesized by other methods and the plant extracts used in the synthesis. ${ }^{35,36}$ These enhanced bioactivities and biocompatibilities can be attributed to the complexation of phytochemicals/secondary metabolites with the nanoparticles. ${ }^{4}$

The potential anticancer, antidiabetic, and antimicrobial applications of green nanoparticles have been discussed in some review articles. ${ }^{37-40}$ To the best of our knowledge, a perspective on the application of nanoparticles synthesized with medicinal plant extracts is not available in the light of Bhasma. By discussing the parallels between Bhasma and green nanoparticles, we argue for the application of the latter in medicine. A schematic representation of the Bhasma preparation and green synthesis of nanoparticles and how these processes lead to the formation of organometallic nanocomplexes is shown in Figure 1.

\section{Use of Plant Products and Metals in Bhasma}

Bhasma preparation is basically done in three steps, namely, 1) shodhana (purification/detoxification), 2) bhavana (purging) and 3) marana (burning). ${ }^{20}$ The impure metal is purified or detoxified with the help of plant and animal products in the first step shodhana. Bhavana is a process of wet grinding in which the purified metal is mixed with a certain liquid medium and ground well. Finally, the ground metal products are dried and packed in a mud pot and burnt several times at high temperature, producing fine ash of the particular metal. ${ }^{20}$ It is believed that the above process of purification, levigation and combustion in the presence of plant and animal products removes impurities and detoxifies the metallic preparations, reduces the size to nano level and increases their effectiveness. ${ }^{25,41,42}$ The particle size of Bhasma was probably stabilized by the calcium present in the plants used, despite repeated burning at high temperatures. ${ }^{43}$ According to the traditional Ayurvedic system of medicine, these Bhasma impart biocompatibility and increased bioavailability to organic molecules from plant extracts. ${ }^{19,21}$

The physico-chemical properties of Bhasma have been characterized by several conventional tests suggested in Ayurvedic textbooks. The physical properties observed are verna (color), nishchandratvam (lustreless), varitara (lightness) and rekhapurnatvam (fineness). The chemical properties tested include apunarbhavata (inability to recover the original metallic form) and niruttha (inability to recover the metallic form). ${ }^{19}$ Recently, many studies reported the properties of Bhasma using advanced scientific methods. Transmission electron 


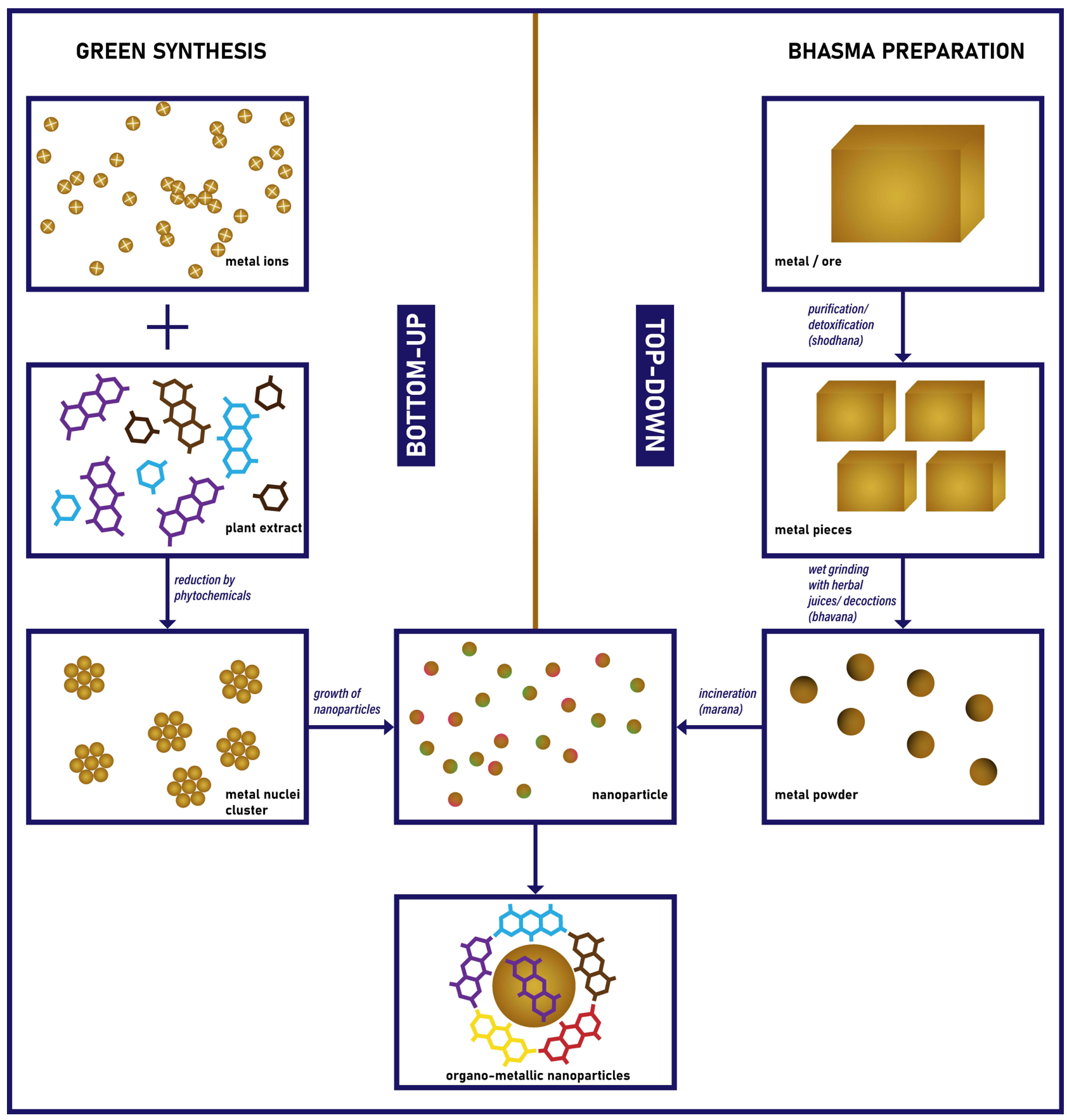

Figure I Schematic presentation of the nano-phytochemical complex formation in green synthesis and bhasma preparation.

microscope (TEM) and scanning electron microscope (SEM) analyses revealed that Vanga Bhasma is polycrystalline and less than $100 \mathrm{~nm}$ in size. ${ }^{22}$ SEM analysis of Krishna Vajra Abhraka Bhasma showed that the Bhasma is square shaped with a mean size of $92.3 \mathrm{~nm} .{ }^{44}$ SEM and X-ray diffraction analysis showed that Swarna Bhasma are large aggregates of smaller nanoparticles of about $60 \mathrm{~nm}$ in size. ${ }^{45}$
Immunomodulation, nontoxicity and the ability to target drugs to the site of action are characteristics of a properly prepared Bhasma. ${ }^{19}$ Medicinal plants used in the various stages of Bhasma preparation are believed to reduce the toxicity of metals (Table 1). Decoction of horse gram used in the purification (shodana) phase of Lauha Bhasma (Fe) preparation removed $\mathrm{Fe}^{3+}$ from the raw material. ${ }^{46}$ Further purification with decoction of three fruits (Phyllanthus 
Table I Medicinal Plants Used in Various Steps of Bhasma Preparation

\begin{tabular}{|c|c|c|c|}
\hline Plant Species Used & Purpose & Bhasma & Reference \\
\hline Vitex negundo L. & \multirow[t]{8}{*}{ Shodhana } & \multirow[t]{2}{*}{ Vanga Bhasma, Yashada Bhasma, Naga Bhasma } & \multirow[t]{2}{*}[22,23,25]{} \\
\hline Curcuma longa $\mathrm{L}$. & & & \\
\hline Citrus jambhiri Lush. & & Tamra Bhasma & [49] \\
\hline Dolichos biflorus L. & & Vaikrānta Bhasma, Swarna Bhasma, Lauha Bhasma & {$[50-52]$} \\
\hline Bauhinia variegata $\mathrm{L}$. & & Swarna Bhasma & {$[50]$} \\
\hline P. emblica & & \multirow[t]{3}{*}{ Lauha Bhasma } & \multirow[t]{3}{*}{ [46] } \\
\hline T. chebula & & & \\
\hline T. bellirica & & & \\
\hline Aloe Vera Tourn. ex Linn. & \multirow[t]{5}{*}{ Bhavana } & Vanga Bhasma & {$[22]$} \\
\hline Amorphophallus campanulatus (Roxb.) Blume ex Decne. & & Tamra Bhasma & [49] \\
\hline Ficus benghalensis $\mathrm{L}$. & & Ras-Sindoor & [53] \\
\hline Plectranthus coesta Buch-Ham & & \multirow[t]{2}{*}{ Naga Bhasma } & \multirow[t]{2}{*}{ [23] } \\
\hline Azadirachta indica A. Juss. & & & \\
\hline Citrus medica L. & \multirow[t]{6}{*}{ Marana } & Vaikrānta Bhasma & {$[52]$} \\
\hline A. Vera & & Yashada Bhasma, Rajata Bhasma & {$[25,54]$} \\
\hline Calotropis procera (Ait.) R.Br. & & \multirow[t]{3}{*}{ Abhraka Bhasma } & \multirow[t]{3}{*}[55]{} \\
\hline Ricinus communis $\mathrm{L}$. & & & \\
\hline Cyperus rotundus $\mathrm{L}$. & & & \\
\hline Adhatoda vasica Nees. & & Naga Bhasma & [23] \\
\hline
\end{tabular}

emblica L. Terminalia chebula Retz. and Terminalia bellirica (Gaertn.) Roxb. kept the $\mathrm{Fe}$ in $\mathrm{Fe}^{2+}$ form and removed any remaining $\mathrm{Fe}^{3+}$ in the sample. ${ }^{46}$ Tinospora cordifolia (Thunb.) Miers (Giloy), known for its efficacy in the treatment of cancer and hypersensitivity due to the presence of isoquinolone alkaloids, was used for the preparation of Kamdudha ras Bhasma - Au and Ag ash. ${ }^{47}$ Similarly, Rosa centifolia L., a plant species with tremendous antioxidant potential ${ }^{48}$ is used for the preparation of $\mathrm{Hg}$ ash/ Kaharva pishti Bhasma. ${ }^{19}$

Bhasma are used in the successful treatment of various types of cancer, autoimmune diseases, tonsillitis, jaundice, anemia, chronic urinary tract infections, ovarian cysts and some other infectious diseases. ${ }^{19,56}$ Some of the Bhasma used in the treatment of different diseases are listed in Table 2.

Yashada $(\mathrm{Zn})$ Bhasma is commonly used in the treatment of diabetes, urinary disorder, eye diseases, etc. ${ }^{25}$
A. vera is used in the marana process of Yashada Bhasma and both $A$. vera and $\mathrm{Zn}$ have the potential to lower blood sugar level. The authors suggest that both metallic $\mathrm{Zn}$ and organic compounds present in $A$. vera may form a coordination bond between them to enhance the medicinal property. ${ }^{25}$ Shankh Bhasma, prepared with $A$. vera and calcium carbonate, is used for stomach-related disorders such as indigestion, flatulence, abdominal pain, vomiting, belching, diarrhea, and gastritis. ${ }^{58}$ It should be noted that calcium carbonate is used in the modern medicine as an antacid to relieve heartburn, acid indigestion, and stomach upset. ${ }^{59} A$. vera is also reported to reduce the symptoms of gastroesophageal reflux disease. ${ }^{60}$ However, no correlation between $A$. vera and calcium carbonate has been experimentally demonstrated for the medical use of Shankh Bhasma. ${ }^{58,61}$ Although organic compounds could be burnt out at higher temperatures used in Bhasma preparations, the presence of organic molecules has been 
Table 2 Some of the Bhasma Preparations Used in the Treatment of Diseases

\begin{tabular}{|c|c|c|c|}
\hline Bhasma & Material Used & Treatment & Reference \\
\hline Abhraka Bhasma & Mica & Chronic cough, anemia and tropical sprue & [55] \\
\hline Lauha Bhasma & $\mathrm{Fe}$ & Hematinic, chronic fever, breathlessness, anti-aging & {$[51,57]$} \\
\hline Naga Bhasma & $\mathrm{Pb}$ & Diarrhea, spleen enlargement and diabetes & [23] \\
\hline Rajata Bhasma & $\mathrm{Ag}$ & Irritable bowel syndrome, acidity, pitta disorders & {$[19,54]$} \\
\hline Ras-Sindoor & $\mathrm{Hg}$ & Syphilis, genital disorders and for rejuvenation & [53] \\
\hline Shankh Bhasma & $\begin{array}{l}\text { Conch shells (Calcium carbonate } \\
\text { or oxide) }\end{array}$ & $\begin{array}{l}\text { Indigestion, flatulence, abdominal pain, vomiting belching, diarrhea, } \\
\text { belching and gastritis }\end{array}$ & [58] \\
\hline Swarna Bhasma & $\mathrm{Au}$ & $\begin{array}{l}\text { Tuberculosis, diabetes mellitus, rheumatoid arthritis and nervous } \\
\text { diseases }\end{array}$ & {$[45,50]$} \\
\hline Swarn makshik Bhasma & Copper pyrite & $\begin{array}{l}\text { Anemia, jaundice, convulsions, insomnia, stomatitis, chronic fever and } \\
\text { skin diseases }\end{array}$ & {$[19,56]$} \\
\hline Tamra Bhasma & $\mathrm{Cu}$ & Ascites, anemia, asthma, hyperacidity & [49] \\
\hline Trivanga Bhasma & $\mathrm{Pb}, \mathrm{Zn}$, and $\mathrm{Sn}$ & Diabetes mellitus and urinary disorders & [24] \\
\hline Vanga Bhasma & $\mathrm{SnO}_{2}$ & $\begin{array}{l}\text { Genitourinary disorder, diabetes, anemia, asthma, gastric ulcers and } \\
\text { urinary diseases }\end{array}$ & [22] \\
\hline Vaikrānta Bhasma & Black tourmaline & Diabetes & {$[19,52]$} \\
\hline Yashada Bhasma & $\mathrm{Zn}$ & Diabetes, eye disorder, urinary disorder & [25] \\
\hline $\begin{array}{l}\text { Krishna Vajra Abhraka } \\
\text { Bhasma }\end{array}$ & Biotite mica & Respiratory diseases & [44] \\
\hline
\end{tabular}

observed in Naga Bhasma and this could be attributed to the organometallic complexes formed at various stages of Bhasma preparation and may be retained even at higher temperatures. ${ }^{23}$ It is believed that Naga Bhasma acts as a carrier for the medicinal properties of various medicinal plants used in different stages of Bhasma preparation such as $V$. negundo, $C$. longa, A. indica and A. vasica. ${ }^{23}$ However, the role of these medicinal plants in the treatment efficacy of Bhashmas in various diseases has not been experimentally validated.

Although Bhasma is used in traditional medicine for centuries to treat various ailments, concerns have been raised about its toxicity. Various steps in the preparation of Bhasma play an important and crucial role in detoxification of metals. After the preparation of Bhasma, it has to undergo a series of tests so that it can be used as medicine. The test for toxicity is called "Varitara" and those Bhasma preparations which have passed the Varitara test are free from toxic metals and can be used as medicine. ${ }^{62}$ Recently, many toxicity studies have been conducted on animal models that showed no toxic effect even at higher therapeutic concentrations. ${ }^{49-51,63-65}$ A study of commercial Ayurvedic formulations (Energic-31 capsule and Basanta Kusumakara Rasa) containing various combinations of different Bhasma showed no adverse/toxic effects in rats. ${ }^{62}$ Similarly, toxicity study of Naga Bhasma and Trivanga Bhasma which contains Lead also showed no toxicity in rats for up to the treatment concentration 60 and $78 \mathrm{mg} / \mathrm{kg}$, respectively. ${ }^{23,24}$ However, administration of higher than therapeutic dose of Tamra Bhasma for 28 days to rats showed mild toxicity in kidney, liver, thymus and heart. ${ }^{49}$ On the other hand, few case reports claim that Bhasma has toxic effects due to higher concentration of metals. ${ }^{66,67}$ A recent case study reported intake of Dahana Bhasma for severe dyspepsia and loss of appetite caused severe liver injury. ${ }^{68}$

\section{Medicinal Plants and Metals in Green Synthesis of Nanoparticles}

Green synthesis is mainly based on the redox process, in which metal ions are first reduced to a cluster of crystallites and then stabilized by the reactive phytochemicals. ${ }^{34,69}$ The 
process of green synthesis involves the preparation of aqueous solutions of metal salt and plant extract and finally mixing. ${ }^{70-72}$ In an aqueous solution, the metal salt dissociates into positive metal ions, which can become supersaturated and form a hydroxyl complex (metal- $\mathrm{OH}$ ). When the plant extract is added slowly to the metal salt solution, the hydroxyl complexes are reduced and then the metal crystallite planes grow. When the crystallite planes are in the higher energy growth phase, secondary metabolites with compatible opposite charge and free activation energy for the reaction would probably come into action and perform a capping action around the high-energy planes. ${ }^{4}$ Several classes of organic compounds have been proposed to be involved in the green synthesis process and a detailed review in this regard has been published recently. ${ }^{4}$ Various classes of plant compounds, namely, alcoholic compounds, terpenoids, quinines, polyphenols (flavones, taxifolin and catechin derivatives), alkaloids, amino acids, polysaccharides, glutathiones, antioxidants, succinic acid, ascorbic acid, tartaric acid, oxalic acid, dihydroxybenzoic acid, etc, were found in the extracts used for green synthesis. ${ }^{4}$ Allicin from Allium sativum L. and curcumin from Curcuma longa L. were successfully used as organic components in the preparation of copper hybrid nanostructures. ${ }^{73,74}$ In addition, heavy molecules such as proteins, glycoproteins and lipids were also found. However, there is evidence in the literature of the involvement of some flavonoids such as rutin, quercetin, etc as reducing or capping agents. ${ }^{4}$ Although many compounds are capable of reducing metal ions to nanoparticles, what is known about whether there is a preference for certain classes of compounds exist, what role these compounds play in forming the nanoparticles, and which compounds in a complex mixture are involved in this process is not clear.

Green synthesized nanoparticles show great promise in pharmacological or therapeutic applications. For example, a number of reports show that green synthesized nanoparticles could fight various types of cancer. $^{40,75-77}$ However, the biological activity of nanoparticles depends on many factors, such as size, shape, capping agent, etc. ${ }^{78,79}$ The presence of plant extract derived capping agents in the nanoparticles may reduce their non-specific toxic behavior. For example, nanoparticles prepared with plant extract were found to be biocompatible for healthy cells, whereas they were lethal for pathogens and tumor growth. $^{80,81}$ Green synthesized $\mathrm{Au}$ nanoparticles with Ocimum tenuiflorum L. extract showed higher anticancer activity than the chemically synthesized Au nanoparticles in several cell lines. ${ }^{81}$ Similarly, Ag nanoparticles synthesized using A. indica extract have less toxic effects against human red blood cells compared to chemically synthesized Ag nanoparticles. ${ }^{82}$ Green synthesised nanoparticles were tested for toxicity in animal models. The silver nanoparticles synthesised using Solanum nigrum L. extract showed lower toxicity to vertebrates and aquatic invertebrates compared to the ionic form of silver. ${ }^{83}$ No adverse or toxic effects on kidney, liver, heart and brain of rats were observed after treatment with silver nanoparticles synthesised using Ficus carica L. fruit extract. ${ }^{84}$ Some of the important medicinal plants used for green synthesis of nanoparticles, their pharmacological properties and corresponding bioactivities of the nanoparticles are listed in Table 3.

Despite a large number of important medicinal plants have been used for green synthesis, many studies have not tested the pharmacological potential of the synthesized nanoparticles. ${ }^{88,96,120,136}$ Moreover, although the extracts of plants with diverse array of medicinal values have been used, the pharmacological properties attributed to these plants/extracts have not been tested in the resulting nanoparticles (Table 3). For example, $\mathrm{ZnO}$ and $\mathrm{Ag}$ nanoparticles synthesized using extracts of $C$. roseus, a well-known medicinal plant in cancer treatment, were not tested for anticancer activities but for antimicrobial activities. ${ }^{100,101}$ Antiplatelet, anti-inflammatory and analgesic activities attributed to $Z$. officinale were not tested in the synthesized Ag nanoparticles. ${ }^{170}$ Similarly, Au nanoparticles synthesized with $R$. communis extract were tested only for anticancer and antimicrobial properties; other pharmacological properties, including prophylactic, purgative, antiinflammatory, and antidiabetic activities, were not tested. ${ }^{158}$ Aqueous extract of $A$. indica leaves has been reported to exhibit significant immunostimulatory activity. ${ }^{172}$ However, three nanoparticles synthesized with the aqueous leaf extract of $A$. indica, namely $\mathrm{Ag}, \mathrm{CeO}_{2}$, and $\mathrm{CuO}$, have not been evaluated for their immunostimulatory activity. ${ }^{94-96}$

Although many studies showed the antioxidant, anticancer and antimicrobial potential of the green synthesized nanoparticles (Table 3), the basis of these properties was not revealed in most of the studies. Although L. nobilis has anticancer activity, how the $\mathrm{ZnO}$ nanoparticles green synthesized with its extract exerted the anticancer activity and the principles of action behind it were not explained. $^{131}$ 
Table 3 The Pharmacological Properties of the Medicinal Plants Used for Green Synthesis of Metal Nanoparticles and the Bioactivities Tested for the Nanoparticles

\begin{tabular}{|c|c|c|c|c|}
\hline Plant Species & Pharmacological Properties of Plant & $\begin{array}{l}\text { Nanoparticles } \\
\text { Synthesized }\end{array}$ & Bioactivities Tested & Reference \\
\hline A. vasica & Antiallergic, antiasthmatic, antiinflammatory, antimicrobial & $\mathrm{Ag}, \mathrm{CuO} / \mathrm{C}$ & Antimicrobial & [85-87] \\
\hline $\begin{array}{l}\text { Agathosma betulina } \\
\text { (Berg.) Pillans }\end{array}$ & Antiseptic, antiinflammatory & $\mathrm{NiO}$ & None tested & [88] \\
\hline $\begin{array}{l}\text { Allium } \\
\text { ampeloprasum L. }\end{array}$ & Antiseptic, antiasthma, diuretic and expectorant & $\mathrm{Ag}$ & Antioxidant & [89] \\
\hline \multirow[t]{2}{*}{ A. vera } & \multirow[t]{2}{*}{ Antimicrobial, antiinflammatory, antiulcer and antiarthritic } & $\mathrm{Ag}$ & Antibacterial & [90] \\
\hline & & $\mathrm{Fe}_{2} \mathrm{O}_{3}$ & None tested & [91] \\
\hline Annona muricata $\mathrm{L}$. & Used in the treatment of cancer and parasitic infections & $\mathrm{Ag}$ & Anticancer & [92] \\
\hline Artemisia annua L. & $\begin{array}{l}\text { Antimicrobial, allelopathic, antiinflammatory, antifeedant and } \\
\text { antitumour }\end{array}$ & $\mathrm{Ag}$ & Antibacterial & [93] \\
\hline \multirow[t]{2}{*}{ A. indica } & \multirow{2}{*}{$\begin{array}{l}\text { Immunostimulant, antibacterial, antioxidant, antiinflammatory } \\
\text { and anticancer }\end{array}$} & $\mathrm{Ag}$ & Antimicrobial & [94] \\
\hline & & $\mathrm{CeO}_{2}, \mathrm{Cu}$ & None tested & {$[95,96]$} \\
\hline $\begin{array}{l}\text { Bergenia ciliata } \\
\text { (Haw.) Sternb. }\end{array}$ & Antitussive, antiulcer, antioxidant, antibacterial and anticancer & $\mathrm{ZnO}$ & $\begin{array}{l}\text { Anticancer and } \\
\text { antibacterial }\end{array}$ & [97] \\
\hline \multirow{2}{*}{$\begin{array}{l}\text { Calotropis gigantea } \\
\text { (L.) Dryand. }\end{array}$} & \multirow{2}{*}{$\begin{array}{l}\text { Purgative, anthelmintic, anticonvulsant, sedative and } \\
\text { antipyretic }\end{array}$} & CdS & Antimicrobial & [98] \\
\hline & & $\mathrm{MgO}$ & None tested & {$[70]$} \\
\hline Carissa carandas L. & $\begin{array}{l}\text { Used in the treatment of scabies, intestinal worms, pruritus, } \\
\text { antiscorbutic, anthelmintic, pain relieving, cancer and } \\
\text { hepatoprotective }\end{array}$ & $\mathrm{Ag}$ & $\begin{array}{l}\text { Antioxidant, anticancer } \\
\text { and antibacterial }\end{array}$ & [99] \\
\hline $\begin{array}{l}\text { Catharanthus roseus } \\
\text { (L.) G. Don }\end{array}$ & $\begin{array}{l}\text { Anticancer, astringent, antibacterial, antidiabetic, antifungal, } \\
\text { and antimalarial }\end{array}$ & $\mathrm{Ag}, \mathrm{ZnO}$ & Antimicrobial & {$[100,101]$} \\
\hline $\begin{array}{l}\text { Catunaregam spinosa } \\
\text { (Thunb.) Tirveng. }\end{array}$ & $\begin{array}{l}\text { Stem bark to treat muscle pain, root bark to treat dandruff, } \\
\text { leaves to cure fever }\end{array}$ & $\mathrm{SnO}_{2}$ & None tested & [7I] \\
\hline $\begin{array}{l}\text { Citrullus colocynthis } \\
\text { (L.) Schrad. }\end{array}$ & Antibacterial, antiinflammatory, antioxidant and anticancer & $\mathrm{ZnO}$ & $\begin{array}{l}\text { Antibacterial, } \\
\text { antioxidant }\end{array}$ & [102] \\
\hline \multirow[t]{2}{*}{ Clitoria ternatea L. } & \multirow{2}{*}{$\begin{array}{l}\text { Diuretic, nootropic, antiasthmatic, anti-inflammatory, } \\
\text { analgesic, antipyretic, antidiabetic, antilipidemic, antiarthritic } \\
\text { and antioxidant }\end{array}$} & $\mathrm{MgO}$ & Antioxidant & [103] \\
\hline & & $\mathrm{SnO}_{2}$ & None tested & [104] \\
\hline \multirow[t]{2}{*}{$\begin{array}{l}\text { Coleus aromaticus } \\
\text { Benth. }\end{array}$} & \multirow[t]{2}{*}{ Antilithic, antispasmodic, cathartic and stimulant } & $\mathrm{Ag}$ & $\begin{array}{l}\text { Antibacterial, } \\
\text { antioxidant }\end{array}$ & [105] \\
\hline & & $\mathrm{Pd}$ & & {$[106]$} \\
\hline $\begin{array}{l}\text { Coptis chinensis } \\
\text { Franch. }\end{array}$ & Antioxidant and antiinflammatory & $\mathrm{Au}$ & Antibacterial & {$[107]$} \\
\hline $\begin{array}{l}\text { Costus speciosus (J. } \\
\text { Koenig) Sm. }\end{array}$ & $\begin{array}{l}\text { Antioxidant, antiinflammatory, antibacterial, antifungal, } \\
\text { analgesic, antipyretic, antidiuretic, larvicidal, and antistress }\end{array}$ & $\mathrm{Ag}$ and $\mathrm{Au}$ & $\begin{array}{l}\text { Antibacterial, } \\
\text { antioxidant }\end{array}$ & {$[108]$} \\
\hline Crocus sativus L. & $\begin{array}{l}\text { Anti-inflammatory, sedative, antiasthma, antioxidant and } \\
\text { adaptogenic agent. }\end{array}$ & $\mathrm{Ag}$ & Antibacterial & [109] \\
\hline
\end{tabular}


Table 3 (Continued).

\begin{tabular}{|c|c|c|c|c|}
\hline Plant Species & Pharmacological Properties of Plant & $\begin{array}{l}\text { Nanoparticles } \\
\text { Synthesized }\end{array}$ & Bioactivities Tested & Reference \\
\hline $\begin{array}{l}\text { Cyclopia intermedia } \\
\text { E. Mey. }\end{array}$ & $\begin{array}{l}\text { Antidiabetic, anticancer, antiobesity, antioxidant and } \\
\text { antimicrobial activities }\end{array}$ & $\mathrm{Au}$ & Anticancer & {$[110]$} \\
\hline $\begin{array}{l}\text { Cynara cardunculus } \\
\text { L. }\end{array}$ & Antioxidant & $\mathrm{Ag}$ & Antibacterial & {$[111]$} \\
\hline C. rotundus & Analgesic, anthelmintic and antifungal activity & $\mathrm{CuO}$ & Antibacterial & {$[112]$} \\
\hline $\begin{array}{l}\text { Dodonaea viscosa } \\
\text { (L.) Jacq }\end{array}$ & $\begin{array}{l}\text { Antibacterial activity, antifungal property, antiviral activity and } \\
\text { antiplasmodial activity. }\end{array}$ & $\mathrm{Ag}$ & $\begin{array}{l}\text { Antibacterial and } \\
\text { anticancer }\end{array}$ & [113] \\
\hline $\begin{array}{l}\text { Eriobotrya japonica } \\
\text { (Thunb.) Lindl. }\end{array}$ & $\begin{array}{l}\text { Anticancer, antioxidant, antiinflammatory, antidiabetic and } \\
\text { antimicrobial }\end{array}$ & $\mathrm{Ag}$ & $\begin{array}{l}\text { Anticancer, antiallergic } \\
\text { and antiinflammatory }\end{array}$ & {$[114]$} \\
\hline $\begin{array}{l}\text { Elephantopus scaber } \\
\text { L. }\end{array}$ & Wound healing, anticancer and antimicrobial & $\mathrm{Ag}$ & $\begin{array}{l}\text { Antioxidant, anticancer } \\
\text { and antimicrobial }\end{array}$ & {$[115]$} \\
\hline $\begin{array}{l}\text { Eleutherococcus } \\
\text { senticosus (Rupr. \& } \\
\text { Maxim.) Maxim. }\end{array}$ & Anticancer, antioxidant, and antimicrobial & $\mathrm{Ag}, \mathrm{Au}$ & $\begin{array}{l}\text { Antioxidant, anticancer } \\
\text { and antibacterial }\end{array}$ & {$[116]$} \\
\hline \multirow{2}{*}{$\begin{array}{l}\text { Eucalyptus globulus } \\
\text { Labill. }\end{array}$} & \multirow{2}{*}{$\begin{array}{l}\text { Antibacterial, antioxidant, analgesic, anti-pyretic and } \\
\text { antiinflammatory }\end{array}$} & $\mathrm{TiO}_{2}, \mathrm{Ag}$ & None tested & {$[117,118]$} \\
\hline & & $\mathrm{ZnO}$ & Antifungal & {$[119]$} \\
\hline \multirow[t]{2}{*}{ F. carica } & \multirow{2}{*}{$\begin{array}{l}\text { Antiinflammatory and antispasmodic; to treat various } \\
\text { ailments such as gastrointestinal, respiratory, and } \\
\text { cardiovascular disorders }\end{array}$} & $\mathrm{Fe}$ & None tested & {$[120]$} \\
\hline & & $\mathrm{Ag}$ & $\begin{array}{l}\text { Anticancer and animal } \\
\text { toxicity }\end{array}$ & {$[84]$} \\
\hline \multirow[t]{3}{*}{ Gloriosa superba L. } & \multirow{3}{*}{$\begin{array}{l}\text { Used as germicide, to cure ulcers, piles, haemorrhoids, } \\
\text { inflammation, scrofula, leprosy, dyspepsia, worm's infestation, } \\
\text { flatulence, intermittent fevers, debility, arthritis, and against } \\
\text { snake poison }\end{array}$} & $\mathrm{Ag}$ and $\mathrm{Au}$ & $\begin{array}{l}\text { Antibacterial, and } \\
\text { antibiofilm }\end{array}$ & {$[121]$} \\
\hline & & $\mathrm{CuO}$ & Antibacterial & {$[122]$} \\
\hline & & Pt and Pd & Anticancer & {$[123]$} \\
\hline Hibiscus sabdariffa L. & Antibacterial and antioxidant & $\mathrm{Au}$ & $\begin{array}{l}\text { Inhibit formation of } \\
\text { amyloid fibrils }\end{array}$ & [124] \\
\hline $\begin{array}{l}\text { Holoptelea } \\
\text { integrifolia (Roxb.) } \\
\text { Planch. }\end{array}$ & $\begin{array}{l}\text { Antioxidant, antibacterial, antimutagenic, antivenom and } \\
\text { antitumor }\end{array}$ & $\mathrm{Ag}$ & $\begin{array}{l}\text { Antioxidant, } \\
\text { antiinflammatory, } \\
\text { antidiabetic and } \\
\text { antibacterial }\end{array}$ & {$[125]$} \\
\hline $\begin{array}{l}\text { Hyptis suaveolens } \\
\text { (L.) Poit. }\end{array}$ & Antirheumatic, antiinflammatory, antifertility & $\mathrm{Ag}$ & Anticancer & [126] \\
\hline $\begin{array}{l}\text { Impatiens balsamina } \\
\text { L. }\end{array}$ & Wound healing & $\mathrm{Ag}$ & Antimicrobial & [127] \\
\hline $\begin{array}{l}\text { Ipomoea asarifolia } \\
\text { (Desr.) Roem. \& } \\
\text { Schult. }\end{array}$ & Diuretic, emmenagogue and purgative & $\mathrm{Ag}$ & Antibacterial & [128] \\
\hline Justicia adhatoda L. & Antiinflammatory, antipyretic & $\mathrm{ZnO}$ & Antimicrobial & [129] \\
\hline
\end{tabular}

(Continued) 
Table 3 (Continued).

\begin{tabular}{|c|c|c|c|c|}
\hline Plant Species & Pharmacological Properties of Plant & $\begin{array}{l}\text { Nanoparticles } \\
\text { Synthesized }\end{array}$ & Bioactivities Tested & Reference \\
\hline Lantana camara L. & Antimicrobial & $\mathrm{Ag}$ & $\begin{array}{l}\text { Antioxidant, anticancer } \\
\text { and antimicrobial }\end{array}$ & {$[127, \mid 30]$} \\
\hline Laurus nobilis L. & Antiseptic, antioxidant, digestive and anticancer & $\mathrm{ZnO}$ & $\begin{array}{l}\text { Anticancer and } \\
\text { antibacterial }\end{array}$ & {$[|3|]$} \\
\hline $\begin{array}{l}\text { Lavandula stoechas } \\
\text { L. }\end{array}$ & Antibacterial, anticholinesterase inhibition and antioxidant & $\mathrm{Ag}$ & $\begin{array}{l}\text { Antibacterial, } \\
\text { antioxidant }\end{array}$ & [132] \\
\hline \multirow{3}{*}{$\begin{array}{l}\text { Matricaria } \\
\text { chamomilla L. }\end{array}$} & \multirow[t]{3}{*}{ Antifungal, anti-inflammatory, antimicrobial and antioxidant } & \multirow[t]{2}{*}{$\mathrm{Ag}$} & Anticancer & [133] \\
\hline & & & Antimicrobial & [134] \\
\hline & & $\mathrm{CuO}$ & $\begin{array}{l}\text { Antioxidant and DNA } \\
\text { cleavage }\end{array}$ & [135] \\
\hline \multirow[t]{2}{*}{ M. pulegium } & \multirow[t]{2}{*}{$\begin{array}{l}\text { Carminative, antitussive, antimicrobial, antioxidant and } \\
\text { antiseptic effects }\end{array}$} & $\mathrm{Ag}$ & $\begin{array}{l}\text { Antimicrobial and } \\
\text { anticancer }\end{array}$ & {$[80]$} \\
\hline & & $\mathrm{Fe}$ & None tested & [136] \\
\hline Morinda citrifolia L. & $\begin{array}{l}\text { Anticancer, analgesic, antimicrobial, and antiinflammatory } \\
\text { activity }\end{array}$ & $\mathrm{Ag}$ & Antibacterial & [137] \\
\hline \multirow{2}{*}{$\begin{array}{l}\text { Moringa oleifera } \\
\text { Lam. }\end{array}$} & \multirow{2}{*}{$\begin{array}{l}\text { Antipyretic, antitumor, antiepileptic, antiulcer, } \\
\text { antiinflammatory, antispasmodic, antihypertensive, diuretic, } \\
\text { antidiabetic, antioxidant and antimicrobial }\end{array}$} & $\mathrm{Pd}, \mathrm{Ag}, \mathrm{Fe}$ & Antimicrobial & {$[138-140]$} \\
\hline & & $\mathrm{Bi}, \mathrm{Cu}$ & $\begin{array}{l}\text { Antimicrobial and } \\
\text { antioxidant }\end{array}$ & {$[141,142]$} \\
\hline Morus alba L. & Antioxidant, hypolipidemic and anti-hyperglycaemic & $\mathrm{Ag}$ & Antibacterial & {$[143]$} \\
\hline $\begin{array}{l}\text { Nervalia zeylanica } \\
\text { (L.) DC. }\end{array}$ & $\begin{array}{l}\text { Cure wounds, colic, ulcers, headache, intestinal worms, } \\
\text { inflammations, rheumatic pain, skin diseases, and leprosy }\end{array}$ & $\mathrm{Ag}$ & $\begin{array}{l}\text { Antimicrobial and } \\
\text { antioxidant }\end{array}$ & {$[144]$} \\
\hline $\begin{array}{l}\text { Mussaenda glabrata } \\
\text { (Hook.f.) Hutch. ex } \\
\text { Gamble }\end{array}$ & Cure ulcers and asthma & $\mathrm{Ag}$ and $\mathrm{Au}$ & Antimicrobial & [145] \\
\hline $\begin{array}{l}\text { Naregamia alata } \\
\text { Wight \& Arn. }\end{array}$ & $\begin{array}{l}\text { Used to cure ulcers, wounds, skin diseases, asthma, arthritis } \\
\text { and fever }\end{array}$ & $\mathrm{Ag}$ and $\mathrm{Au}$ & Antimicrobial & {$[146]$} \\
\hline \multirow[t]{2}{*}{ Ocimum sanctum L. } & \multirow{2}{*}{$\begin{array}{l}\text { Antioxidant, antibiotic, antidiabetic, antiatherogenic, } \\
\text { immunomodulatory, antiinflammatory, analgesic, antiulcer, } \\
\text { chemo-preventive and antipyretic }\end{array}$} & $\mathrm{Ag}$ & Antibacterial & [147] \\
\hline & & $\mathrm{Ni}$ & None tested & {$[148]$} \\
\hline Olea europaea L. & $\begin{array}{l}\text { Antimalarial, antibacterial, antioxidant, antiinflammatory and } \\
\text { antimycoplasma }\end{array}$ & $\mathrm{Ag}$ & $\begin{array}{l}\text { Anticancer and } \\
\text { antibacterial }\end{array}$ & [149] \\
\hline $\begin{array}{l}\text { Pelargonium } \\
\text { endlicherianum } \\
\text { Fenzl. }\end{array}$ & $\begin{array}{l}\text { Used to treat cough, sore throat, congestion and other } \\
\text { respiratory ailments }\end{array}$ & $\mathrm{Ag}$ & Antimicrobial & {$[150]$} \\
\hline $\begin{array}{l}\text { Phyla dulcis (Trevir.) } \\
\text { Moldenke }\end{array}$ & Antiproliferative and antiviral & $\mathrm{Ag}$ & Antimicrobial & {$[151]$} \\
\hline
\end{tabular}

(Continued) 
Table 3 (Continued).

\begin{tabular}{|c|c|c|c|c|}
\hline Plant Species & Pharmacological Properties of Plant & $\begin{array}{l}\text { Nanoparticles } \\
\text { Synthesized }\end{array}$ & Bioactivities Tested & Reference \\
\hline \multirow[t]{2}{*}{ P. emblica } & \multirow[t]{2}{*}{$\begin{array}{l}\text { Antioxidant activity, antiaging, antipuretic, and } \\
\text { antiinflammatory }\end{array}$} & Pd & $\begin{array}{l}\text { Anticancer and } \\
\text { antibacterial }\end{array}$ & {$[152]$} \\
\hline & & $\mathrm{Ag}$ & Antibacterial & [153] \\
\hline Phyllanthus Niruri L. & $\begin{array}{l}\text { Used in the treatment of jaundice, diarrhea, kidney aliments, } \\
\text { constipation, ulcers, malaria, hemorrhoids, ringworm and } \\
\text { hemorrhoids }\end{array}$ & $\mathrm{TiO}_{2}$ & None tested & {$[154]$} \\
\hline Pimpinella anisum L. & $\begin{array}{l}\text { Antioxidant, antibacterial, antifungal, anticonvulsant, } \\
\text { antiinflammatory, analgesic, gastro-protective, antidiabetic, } \\
\text { and antiviral activities }\end{array}$ & $\mathrm{Ag}$ & $\begin{array}{l}\text { Anticancer and } \\
\text { antibacterial }\end{array}$ & {$[155]$} \\
\hline Piper nigrum L. & $\begin{array}{l}\text { Antipyretic, analgesic, hepatoprotective, antioxidant and anti- } \\
\text { inflammatory }\end{array}$ & $\mathrm{Ag}$ & $\begin{array}{l}\text { Antioxidant and } \\
\text { toxicity in animal }\end{array}$ & [156] \\
\hline Pongamia pinnata $\mathrm{L}$. & Anti-inflammatory, analgesic & $\mathrm{Au}$ & Antifungal & {$[157]$} \\
\hline R. communis & $\begin{array}{l}\text { Prophylactic, purgative, antimicrobial, antiinflammatory and } \\
\text { antidiabetic }\end{array}$ & $\mathrm{Au}$ & $\begin{array}{l}\text { Anticancer and } \\
\text { antimicrobial }\end{array}$ & {$[158]$} \\
\hline Rosa canina L. & Anti-inflammatory, antioxidants & $\mathrm{ZnO}$ & $\begin{array}{l}\text { Anticancer and } \\
\text { antibacterial }\end{array}$ & [159] \\
\hline Saraca indica $\mathrm{L}$. & $\begin{array}{l}\text { To cure gynaecological disorders like pelvic pain, } \\
\text { endometriosis, menorrhagia, uterine fibroids, haemorrhagic } \\
\text { dysentery }\end{array}$ & $\mathrm{Ag}$ & Antimicrobial & {$[160]$} \\
\hline \multirow[t]{2}{*}{ Salvadora persica $\mathrm{L}$. } & \multirow[t]{2}{*}{ Abrasive, astringent, antiseptic and detergent } & $\mathrm{Ag}$ & Antimicrobial & {$[161,162]$} \\
\hline & & $\mathrm{ZnO}$ & Anticancer & [163] \\
\hline S. nigrum & Used for treating inflammation, pain and fever & $\mathrm{Ag}$ & Toxicity in animals & {$[83]$} \\
\hline $\begin{array}{l}\text { Stereospermum } \\
\text { suaveolens (Roxb.) } \\
\text { DC. }\end{array}$ & $\begin{array}{l}\text { Used for treating vomiting, fever, asthma, blood and heart } \\
\text { infections }\end{array}$ & $\mathrm{Ag}$ and $\mathrm{Au}$ & $\begin{array}{l}\text { Antioxidant, anticancer } \\
\text { and antibacterial }\end{array}$ & {$[164]$} \\
\hline $\begin{array}{l}\text { Syzygium } \\
\text { aromaticum (L.) } \\
\text { Merr. \& L.M.Perry }\end{array}$ & Antiseptic, antibacterial, antifungal and antiviral & $\mathrm{Ag}$ & Antimicrobial & {$[165]$} \\
\hline T. bellirica & Laxative, astringent, anthelmintic and antipyretic & $\mathrm{Ag}$ & Antimicrobial & [166] \\
\hline T. cordifolia & $\begin{array}{l}\text { Anticancer, antileprotic, antihyperglycemic, antiallergic and } \\
\text { antidiabetic }\end{array}$ & $\mathrm{Ag}$ & $\begin{array}{l}\text { Antioxidant and } \\
\text { antibacterial }\end{array}$ & {$[167]$} \\
\hline $\begin{array}{l}\text { Withania coagulans } \\
\text { (Stocks) Dunal }\end{array}$ & $\begin{array}{l}\text { Antihyperglycaemic, anti hypercholesterolemic, antifungal, } \\
\text { antibacterial and anticancer }\end{array}$ & $\mathrm{Ag}$ & $\begin{array}{l}\text { Antioxidant, anticancer } \\
\text { and antibacterial }\end{array}$ & {$[168]$} \\
\hline \multirow[t]{2}{*}{$\begin{array}{l}\text { Withania somnifera } \\
\text { (L.) Dunal }\end{array}$} & \multirow[t]{2}{*}{ Antioxidant, antilnflammatory, antitumor and antistress } & $\mathrm{Se}$ & $\begin{array}{l}\text { Antioxidant, anticancer } \\
\text { and antibacterial }\end{array}$ & [169] \\
\hline & & $\mathrm{Ag}$ & Antimicrobial & {$[72]$} \\
\hline $\begin{array}{l}\text { Zingiber officinale } \\
\text { Roscoe }\end{array}$ & Antiplatelet, antiinflammatory and analgesic & $\mathrm{Ag}$ & Antibacterial & {$[170]$} \\
\hline $\begin{array}{l}\text { Ziziphus zizyphus } \\
\text { (L.) H. Karst. }\end{array}$ & Antifungal, antibacterial, antiulcer, and antiinflammatory & $\mathrm{Au}$ & Antimicrobial & {$[|7|]$} \\
\hline
\end{tabular}




\section{Conclusion}

The organometallic nature and biocompatibility of the green synthesized nanoparticles indicate the possibility of their application as ideal candidates for the development of phytonanomedicines. Despite a large number of medicinal plants with important pharmacological properties were used in green synthesis, most studies have not tested the pharmacological properties attributed to those plants/extracts or the bioactivity of the nanoparticles at all, making it difficult to establish a correlation between the medicinal plants used in the process and the nanoparticles. Therefore, testing the pharmacological properties corresponding to the plant extracts in green synthesized nanoparticles would be very useful to advance this field of nanomedicine. Similarly, comparative analyses of the pharmacological properties of green synthesized nanoparticles and Bhasma prepared using the same plants/extracts would provide new clues. Since green synthesis imparts novel pharmacological properties to the resulting nanoparticles, a clear understanding of the specific compounds involved in the reduction and capping processes would further advance this area of research. Detailed toxicity studies are required before green synthesized nanoparticles are considered for medical applications.

\section{Acknowledgments}

This work was supported by the National Science Centre (NCN), OPUS project (Reg. No 2016/21/B/ NZ9/01980). RKS received a postdoctoral fellowship from this project.

\section{Disclosure}

The authors report no conflicts of interest in this work.

\section{References}

1. Marslin G, Sheeba CJ, Franklin G. Nanoparticles alter secondary metabolism in plants via ROS Burst. Front Plant Sci. 2017;8:832. doi:10.3389/fpls.2017.00832

2. Slavin YN, Asnis J, Häfeli UO, Bach H. Metal nanoparticles: understanding the mechanisms behind antibacterial activity. J Nanobiotechnology. 2017;15(1):65. doi:10.1186/s12951-017-0308-Z

3. Luther DC, Huang R, Jeon T, et al. Delivery of drugs, proteins, and nucleic acids using inorganic nanoparticles. Adv Drug Deliv Rev. 2020;156:188-213. doi:10.1016/j.addr.2020.06.020

4. Marslin G, Siram K, Maqbool Q, et al. Secondary metabolites in the green synthesis of metallic nanoparticles. Materials. 2018;11(6):940. doi:10.3390/ma11060940

5. de Lázaro I, Mooney DJ. Obstacles and opportunities in a forward vision for cancer nanomedicine. Nat Mater. 2021. doi:10.1038/s41563021-01047-7
6. Jiang W, Wang Y, Wargo JA, Lang FF, Kim BYS. Considerations for designing preclinical cancer immune nanomedicine studies. Nat Nanotechnol. 2021;16(1):6-15. doi:10.1038/s41565-02000817-9

7. Barabadi H, Alizadeh Z, Rahimi MT, et al. Nanobiotechnology as an emerging approach to combat malaria: a systematic review. Nanomed Nanotechnol Biol Med. 2019;18:221-233. doi:10.1016/ j.nano.2019.02.017

8. Connor EE, Mwamuka J, Gole A, Murphy CJ, Wyatt MD. Gold Nanoparticles Are Taken Up by Human Cells but Do Not Cause Acute Cytotoxicity. Small. 2005;1(3):325-327. doi:10.1002/ smll.200400093

9. Sousa de Almeida M, Susnik E, Drasler B, Taladriz-Biianco P, Petri-Frank A, Rothen-Rutishauser B. Understanding nanoparticle endocytosis to improve targeting strategies in nanomedicine. Chem Soc Rev. 2021;50(9):5397-5434. doi:10.1039/ D0CS01127D

10. Sindhwani S, Chan WCW. Nanotechnology for modern medicine: next step towards clinical translation. J Intern Med. 2021;290 (3):486-498. doi:10.1111/joim.13254

11. Nelaturi P, Nagarajan P, Sabapathy SK, Sambandam R. Swarna Bindu Prashana - an Ancient Approach to Improve the Infant's Immunity. Biol Trace Elem Res. 2021;199(6):2145-2148. doi:10.1007/s12011-020-02353-y

12. Trujillo-Alonso V, Pratt EC, Zong H, et al. FDA-approved ferumoxytol displays anti-leukaemia efficacy against cells with low ferroportin levels. Nat Nanotechnol. 2019;14(6):616-622. doi:10.1038/s41565-019-0406-1

13. Strauss WE, Dahl NV, Li Z, Lau G, Allen LF. Ferumoxytol versus iron sucrose treatment: a post-hoc analysis of randomized controlled trials in patients with varying renal function and iron deficiency anemia. BMC Hematol. 2016;16(1):20. doi:10.1186/ s12878-016-0060-x

14. He Y, Tian F, Zhou J, Zhao Q, Fu R, Jiao B. Colorimetric aptasensor for ochratoxin A detection based on enzyme-induced gold nanoparticle aggregation. $J$ Hazard Mater. 2020;388:121758. doi:10.1016/j.jhazmat.2019.121758

15. Meng Z, Huang H, Huang D, Zhang F, Mi P. Functional metalorganic framework-based nanocarriers for accurate magnetic resonance imaging and effective eradication of breast tumor and lung metastasis. $J$ Colloid Interface Sci. 2021;581:31-43. doi:10.1016/j.jcis.2020.07.072

16. Sulaiman GM, Waheeb HM, Jabir MS, Khazaal SH, Dewir YH, Naidoo Y. Hesperidin Loaded on Gold Nanoparticles as a Drug Delivery System for a Successful Biocompatible, Anti-Cancer, Anti-Inflammatory and Phagocytosis Inducer Model. Sci Rep. 2020;10(1):9362. doi:10.1038/s41598-020-66419-6

17. Jabir M, Sahib UI, Taqi Z, et al. Linalool-loaded glutathionemodified gold nanoparticles conjugated with calnn peptide as apoptosis inducer and nf- $\mathrm{kb}$ translocation inhibitor in skov-3 cell line. Int J Nanomedicine. 2020;15:9025-9047. doi:10.2147/ IJN.S276714

18. Balkrishna A, Solleti SK, Singh H, Tomer M, Sharma N, Varshney A. Calcio-herbal formulation, Divya-Swasari-Ras, alleviates chronic inflammation and suppresses airway remodelling in mouse model of allergic asthma by modulating pro-inflammatory cytokine response. Biomed Pharmacother. 2020;126:110063. doi:10.1016/j.biopha.2020.110063

19. Pal D, Sahu CK, Haldar A. Bhasma: the ancient Indian nanomedicine. $J$ Adv Pharm Technol Res. 2014;5(1):4-12. doi:10.4103/2231-4040.126980

20. Sarkar PK, Chaudhary AK. Ayurvedic bhasma: the most ancient application of nanomedicine. $J$ Sci Ind Res. 2010;69 (12):901-905. 
21. Kamath SU, Pemiah B, Sekar RK, Krishnaswamy S, Sethuraman S, Krishnan UM. Mercury-based traditional herbo-metallic preparations: a toxicological perspective. Arch Toxicol. 2012;86(6):831-838. doi:10.1007/s00204-012-0826-2

22. Kale B, Rajurkar N. Synthesis and characterization of Vanga bhasma. $J$ Ayurveda Integr Med. 2019;10(2):111-118. doi:10.1016/j.jaim.2017.05.003

23. Singh SK, Gautam DNS, Kumar M, Rai SB. Synthesis, characterization and histopathological study of a lead-based Indian traditional drug: naga bhasma. Indian J Pharm Sci. 2010;72 (1):24-30. doi:10.4103/0250-474X.62232

24. Jamadagni PS, Jamadagni SB, Singh R, Gaidhani SN, Upadhyay S, Hazra J. Repeated dose oral toxicity of Trivanga Bhasma in Swiss albino mice. Ayu. 2013;34(1):118-123. doi:10.4103/0974-8520.115449

25. Pareek A, Bhatnagar N. Physico-chemical characterization of traditionally prepared Yashada bhasma. J Ayurveda Integr Med. 2020;11(3):228-235. doi:10.1016/j.jaim.2018.11.004

26. Injal AS. Are Bhasma Nanomedicine of Ancient Times. Hist Nanotechnol. 2019;57-89. doi:10.1002/9781119460534.ch4

27. Ocsoy I, Gulbakan B, Chen T, et al. DNA-Guided Metal-Nanoparticle Formation on Graphene Oxide Surface. $A d v$ Mater. 2013;25(16):2319-2325. doi:10.1002/adma.201204944

28. Leng Y, Fu L, Ye L, et al. Protein-directed synthesis of highly monodispersed, spherical gold nanoparticles and their applications in multidimensional sensing. Sci Rep. 2016;6(1):28900. doi:10.1038/srep28900

29. Barabadi H, Tajani B, Moradi M, et al. Penicillium family as emerging nanofactory for biosynthesis of green nanomaterials: a journey into the world of microorganisms. J Clust Sci. 2019;30(4):843-856. doi:10.1007/s10876-019-01554-3

30. Moreno-Luna FB, Tovar-Corona A, Herrera-Perez JL, SantoyoSalazar J, Rubio-Rosas E, Vázquez-Cuchillo O. Quick synthesis of gold nanoparticles at low temperature, by using Agave potatorum extracts. Mater Lett. 2019;235:254-257. doi:10.1016/j. matlet.2018.09.122

31. Garibo D, Borbón-Nuñez HA, de León JND, et al. Green synthesis of silver nanoparticles using Lysiloma acapulcensis exhibit high-antimicrobial activity. Sci Rep. 2020;10(1):12805. doi:10.1038/s41598-020-69606-7

32. Philip D. Rapid green synthesis of spherical gold nanoparticles using Mangifera indica leaf. Spectrochim Acta Part A Mol Biomol Spectrosc. 2010;77(4):807-810. doi:10.1016/j.saa.2010.08.008

33. Vanlalveni C, Lallianrawna S, Biswas A, Selvaraj M, Changmai B, Rokhum SL. Green synthesis of silver nanoparticles using plant extracts and their antimicrobial activities: a review of recent literature. RSC $A d v$. 2021;11(5):2804-2837. doi:10.1039/ D0RA09941D

34. Chandra H, Kumari P, Bontempi E, Yadav S. Medicinal plants: treasure trove for green synthesis of metallic nanoparticles and their biomedical applications. Biocatal Agric Biotechnol. 2020;24:101518. doi:10.1016/j.bcab.2020.101518

35. Augustine R, Hasan A. Emerging applications of biocompatible phytosynthesized metal/metal oxide nanoparticles in healthcare. J Drug Deliv Sci Technol. 2020;56:101516. doi:10.1016/j. jddst.2020.101516

36. Sudhasree S, Shakila Banu A, Brindha P, Kurian GA. Synthesis of nickel nanoparticles by chemical and green route and their comparison in respect to biological effect and toxicity. Toxicol Environ Chem. 2014;96(5):743-754. doi:10.1080/ 02772248.2014.923148

37. Singh A, Gautam PK, Verma A, et al. Green synthesis of metallic nanoparticles as effective alternatives to treat antibiotics resistant bacterial infections: a review. Biotechnol Reports. 2020;25: e00427. doi:10.1016/j.btre.2020.e00427
38. Nadeem M, Khan R, Afridi K, et al. Green synthesis of cerium oxide nanoparticles $(\mathrm{CeO}(2) \mathrm{NPs})$ and their antimicrobial applications: a review. Int J Nanomedicine. 2020;15:5951-5961. doi:10.2147/IJN.S255784

39. Anand K, Tiloke C, Naidoo P, Chuturgoon AA. Phytonanotherapy for management of diabetes using green synthesis nanoparticles. J Photochem Photobiol B Biol. 2017;173:626-639. doi:10.1016/j. jphotobiol.2017.06.028

40. Khatua A, Prasad A, Priyadarshini E, et al. Emerging antineoplastic plant-based gold nanoparticle synthesis: a mechanistic exploration of their anticancer activity toward cervical cancer cells. J Clust Sci. 2020;31(6):1329-1340. doi:10.1007/s10876019-01742-1

41. Liu J, Zhang F, Ravikanth V, Olajide OA, Li C, Wei L-X. Chemical compositions of metals in Bhasmas and Tibetan Zuotai are a major determinant of their therapeutic effects and toxicity. Evidence-Based Complement Altern Med. 2019;2019:1697804. doi:10.1155/2019/1697804

42. Chaudhary A. Ayurvedic Bhasma: nanomedicine of ancient India - Its global contemporary perspective. J Biomed Nanotechnol. 2011;7(1):68-69. doi:10.1166/jbn.2011.1205

43. Wadekar MP, Rode CV, Bendale YN, Patil KR, Gaikwad AB, Prabhune AA. Effect of calcination cycles on the preparation of tin oxide based traditional drug: studies on its formation and characterization. J Pharm Biomed Anal. 2006;41(4):1473-1478. doi:10.1016/j.jpba.2006.03.032

44. Wele A, De S, Dalvi M, Devi N, Pandit V. Nanoparticles of biotite mica as Krishna Vajra Abhraka Bhasma: synthesis and characterization. J Ayurveda Integr Med. 2021;12(2):269-282. doi:10.1016/j.jaim.2020.09.004

45. Beaudet D, Badilescu S, Kuruvinashetti K, et al. Comparative study on cellular entry of incinerated ancient gold particles (Swarna Bhasma) and chemically synthesized gold particles. Sci Rep. 2017;7(1):10678. doi:10.1038/s41598-017-10872-3

46. Krishnamachary B, Rajendran N, Pemiah B, et al. Scientific validation of the different purification steps involved in the preparation of an Indian Ayurvedic medicine, Lauha bhasma. $J \quad$ Ethnopharmacol. 2012;142(1):98-104. doi:10.1016/j. jep.2012.04.021

47. Saha S, Ghosh S. Tinospora cordifolia: one plant, many roles. Anc Sci Life. 2012;31(4):151-159. doi:10.4103/0257-7941.107344

48. Kumar S, Gautam S, Powar S, Sharma A. Microbial decontamination of medicinally important herbals using gamma radiation and their biochemical characterisation. Food Chem. 2010;119 (1):328-335. doi:10.1016/j.foodchem.2009.06.034

49. Chaudhari SY, Nariya MB, Galib R, Prajapati PK. Acute and subchronic toxicity study of Tamra Bhasma (incinerated copper) prepared with and without Amritikarana. J Ayurveda Integr Med. 2016;7(1):23-29. doi:10.1016/j.jaim.2015.11.001

50. Biswas S, Dhumal R, Selkar N, et al. Physicochemical characterization of Suvarna Bhasma, its toxicity profiling in rat and behavioural assessment in zebrafish model. $J$ Ethnopharmacol. 2020;249:112388. doi:10.1016/j.jep.2019.112388

51. Joshi N, Dash MK, Dwivedi L, Khilnani GD. Toxicity study of Lauha Bhasma (calcined iron) in albino rats. Anc Sci Life. 2016;35(3):159-166. doi:10.4103/0257-7941.179870

52. Tripathi R, Rathore AS, Mehra BL, Raghubir R. Physicochemical study of Vaikrānta bhasma. Anc Sci Life. 2013;32 (4):199-204. doi:10.4103/0257-7941.131971

53. Singh SK, Chaudhary A, Rai DK, Rai SB. Preparation and characterization of a mercury based Indian traditional drugRas-Sindoor. Indian J Tradit Knowl. 2009;8(3):346-351.

54. Chaturvedi R, Jha CB. Standard manufacturing procedure of Rajata Bhasma. Ayu. 2011;32(4):566-571. doi:10.4103/09748520.96135 
55. Kantak S, Rajurkar N, Adhyapak P. Synthesis and characterization of Abhraka (mica) bhasma by two different methods. J Ayurveda Integr Med. 2019. doi:10.1016/j.jaim.2018.11.003

56. Mohaptra S, Jha CB. Physicochemical characterization of Ayurvedic bhasma (Swarna makshika bhasma): an approach to standardization. Int $J$ Ayurveda Res. 2010;1(2):82-86. doi:10.4103/0974-7788.64409

57. Singh N, Reddy KRC. Pharmaceutical study of Lauha Bhasma. Ayu. 2010;31(3):387-390. doi:10.4103/0974-8520.77157

58. Singh RK, Kumar S, Aman AK, Kr S, Kar M, Kumar S. Physical properties of an Indian Ayurvedic medicine (Shankh Bhasma) as nano materials for its application. Indian J Tradit Knowl. 2019;18 (1):178-183.

59. Voropaiev M, Nock D. Onset of acid-neutralizing action of a calcium/magnesium carbonate-based antacid using an artificial stomach model: an in vitro evaluation. BMC Gastroenterol. 2021;21(1):112. doi:10.1186/s12876-021-01687-8

60. Panahi Y, Khedmat H, Valizadegan G, Mohtashami R, Sahebkar A. Efficacy and safety of Aloe vera syrup for the treatment of gastroesophageal reflux disease: a pilot randomized positive-controlled trial. $J$ Tradit Chinese Med. 2015;35 (6):632-636. doi:10.1016/S0254-6272(15)30151-5

61. Chavan S, Tayade S, Gupta V, Deshmukh V, Sardeshmukh S. Pharmaceutical standardization and physicochemical characterization of traditional ayurvedic marine drug: incinerated conch shell (shankha bhasma). Mar Drugs. 2018;16:11. doi:10.3390/ md16110450

62. Kumar G, Gupta Y. Evidence for safety of Ayurvedic herbal, herbo-metallic and Bhasma preparations on neurobehavioral activity and oxidative stress in rats. AYU. 2012;33(4):569-575. doi:10.4103/0974-8520.110514

63. Nagaraju V, Joshi D, Aryya NC. Toxicity studies on vanga bhasma (Part I - with special reference to G. I. T. Liver and Pancreas). Anc Sci Life. 1984;4(1):32-35.

64. Gokarn RA, Nariya MB, Patgiri BJ, Prajapati PK. Toxicological studies of Rasasindura, an ayurvedic formulation. Indian J Pharm Sci. 2017;79(4):633-640. doi:10.4172/pharmaceutical-sciences. 1000272

65. Umrani RD, Paknikar KM. Ayurvedic medicine zinc bhasma: physicochemical evaluation, anti-diabetic activity and safety assessment. J Biomed Nanotechnol. 2011;7(1):148-149. doi:10.1166/jbn.2011.1243

66. Raviraja A, Vishal Babu GN, Sehgal A, et al. Three cases of lead toxicity associated with consumption of ayurvedic medicines. Indian J Clin Biochem. 2010;25(3):326-329. doi:10.1007/ s12291-010-0051-9

67. Breeher L, Gerr F, Fuortes L. A case report of adult lead toxicity following use of Ayurvedic herbal medication. $J$ Occup Med Toxicol. 2013;8(1):26. doi:10.1186/1745-6673-8-26

68. Philips CA, Paramaguru R, Augustine P. Ayurveda metallicmineral 'Bhasma'-associated severe liver injury. BMJ Case Rep. 2018;2018:bcr-2018-225590. doi:10.1136/bcr-2018-225590

69. Singh J, Dutta T, Kim K-H, Rawat M, Samddar P, Kumar P. 'Green' synthesis of metals and their oxide nanoparticles: applications for environmental remediation. $J$ Nanobiotechnology. 2018;16(1):84. doi:10.1186/s12951-018-0408-4

70. Hii YS, Jeevanandam J, Chan YS. Plant mediated green synthesis and nanoencapsulation of $\mathrm{MgO}$ nanoparticle from Calotropis gigantea: characterisation and kinetic release studies. Inorg NanoMetal Chem. 2018;48(12):620-631. doi:10.1080/ 24701556.2019.1569053

71. Haritha E, Roopan SM, Madhavi G, Elango G, Al-Dhabi NA, Arasu MV. Green chemical approach towards the synthesis of $\mathrm{SnO} 2 \mathrm{NPs}$ in argument with photocatalytic degradation of diazo dye and its kinetic studies. J Photochem Photobiol B Biol. 2016;162:441-447. doi:10.1016/j.jphotobiol.2016.07.010
72. Marslin G, Selvakesavan RK, Franklin G, Sarmento B, Dias ACP. Antimicrobial activity of cream incorporated with silver nanoparticles biosynthesized from Withania somnifera. Int J Nanomedicine. 2015;10:5955-5963. doi:10.2147/IJN.S81271

73. Koca FD, Demirezen Yilmaz D, Ertas Onmaz N, Yilmaz E, Ocsoy I. Green synthesis of allicin based hybrid nanoflowers with evaluation of their catalytic and antimicrobial activities. Biotechnol Lett. 2020;42(9):1683-1690. doi:10.1007/s10529-020-02877-2

74. Koca FD, Demirezen Yilmaz D, Ertas Onmaz N, Ocsoy I. Peroxidase-like activity and antimicrobial properties of curcumin-inorganic hybrid nanostructure. Saudi J Biol Sci. 2020;27(10):2574-2579. doi:10.1016/j.sjbs.2020.05.025

75. Saravanan M, Barabadi H, Ramachandran B, Venkatraman G, Ponmurugan K. Chapter Eleven - Emerging plant-based anticancer green nanomaterials in present scenario. In: Verma SK, Das AKBT-CA-C, editors. Engineered Nanomaterials and Phytonanotechnology: Challenges for Plant Sustainability. Vol. 87. Elsevier; 2019:291-318.

76. Abdellatif AAH, Alsharidah M, Al Rugaie O, Tawfeek HM, Tolba NS. Silver nanoparticle-coated ethyl cellulose inhibits tumor necrosis factor- $\alpha$ of breast cancer cells. Drug Des Devel Ther. 2021;15:2035-2046. doi:10.2147/DDDT.S310760

77. Joseph J, Khor KZ, Moses EJ, Lim V, Aziz MY, Abdul Samad N. In vitro anticancer effects of Vernonia amygdalina leaf extract and green-synthesised silver nanoparticles. Int $J$ Nanomedicine. 2021;16:3599-3612. doi:10.2147/IJN.S303921

78. Barabadi H, Damavandi Kamali K, Jazayeri Shoushtari F, et al. Emerging theranostic silver and gold nanomaterials to combat prostate cancer: a systematic review. J Clust Sci. 2019;30 (6):1375-1382. doi:10.1007/s10876-019-01588-7

79. Rónavári A, Kovács D, Igaz N, et al. Biological activity of green-synthesized silver nanoparticles depends on the applied natural extracts: a comprehensive study. Int $J$ Nanomedicine. 2017;12:871-883. doi:10.2147/IJN.S122842

80. Kelkawi AHA, Abbasi Kajani A, Bordbar A-K. Green synthesis of silver nanoparticles using Mentha pulegium and investigation of their antibacterial, antifungal and anticancer activity. IET Nanobiotechnology. 2017;11(4):370-376. doi:10.1049/iet-nbt. 2016.0103

81. Virmani I, Sasi C, Priyadarshini E, et al. Comparative anticancer potential of biologically and chemically synthesized gold nanoparticles. J Clust Sci. 2020;31(4):867-876. doi:10.1007/ s10876-019-01695-5

82. Kummara S, Patil MB, Uriah T. Synthesis, characterization, biocompatible and anticancer activity of green and chemically synthesized silver nanoparticles - a comparative study. Biomed Pharmacother. 2016;84:10-21. doi:10.1016/j.biopha.2016.09.003

83. Jenifer AA, Malaikozhundan B, Vijayakumar S, Anjugam M, Iswarya A, Vaseeharan B. Green synthesis and characterization of silver nanoparticles (AgNPs) using leaf extract of Solanum nigrum and assessment of toxicity in vertebrate and invertebrate aquatic animals. J Clust Sci. 2020;31(5):989-1002. doi:10.1007/ s10876-019-01704-7

84. Jacob SJP, Prasad VLS, Sivasankar S, Muralidharan P. Biosynthesis of silver nanoparticles using dried fruit extract of Ficus carica - Screening for its anticancer activity and toxicity in animal models. Food Chem Toxicol. 2017;109:951-956. doi:10.1016/j.fct.2017.03.066

85. Latha M, Priyanka M, Rajasekar P, Manikandan R, Prabhu NM. Biocompatibility and antibacterial activity of the Adathoda vasica Linn extract mediated silver nanoparticles. Microb Pathog. 2016;93:88-94. doi:10.1016/J.MICPATH.2016.01.013

86. Bhavyasree PG, Xavier TS. Green synthesis of copper oxide/ carbon nanocomposites using the leaf extract of Adhatoda vasica Nees, their characterization and antimicrobial activity. Heliyon. 2020;6(2):e03323-e03323. doi:10.1016/j.heliyon.2020.e03323 
87. Thirumagal N, Jeyakumari AP. Structural, optical and antibacterial properties of green synthesized silver nanoparticles (AgNPs) using Justicia adhatoda L. J Clust Sci. 2020;31(2):487-497. doi:10.1007/s10876-019-01663-z

88. Thema FT, Manikandan E, Gurib-Fakim A, Maaza M. Single phase Bunsenite $\mathrm{NiO}$ nanoparticles green synthesis by Agathosma betulina natural extract. $J$ Alloys Compd. 2016;657:655-661. doi:10.1016/j.jallcom.2015.09.227

89. Khoshnamvand M, Huo C, Liu J. Silver nanoparticles synthesized using Allium ampeloprasum L. leaf extract: characterization and performance in catalytic reduction of 4-nitrophenol and antioxidant activity. J Mol Struct. 2019;1175:90-96. doi:10.1016/j. molstruc.2018.07.089

90. Tippayawat P, Phromviyo N, Boueroy P, Chompoosor A. Green synthesis of silver nanoparticles in aloe vera plant extract prepared by a hydrothermal method and their synergistic antibacterial activity. PeerJ. 2016;4:e2589-e2589. doi:10.7717/peerj.2589

91. Chandrababu P, Cheriyan S, Raghavan R. Aloe vera leaf extract-assisted facile green synthesis of amorphous $\mathrm{Fe} 2 \mathrm{O} 3$ for catalytic thermal decomposition of ammonium perchlorate. J Therm Anal Calorim. 2020;139(1):89-99. doi:10.1007/s10973019-08376-5

92. Jabir MS, Saleh YM, Sulaiman GM, et al. Green synthesis of silver nanoparticles using annona muricata extract as an inducer of apoptosis in cancer cells and inhibitor for NLRP3 inflammasome via enhanced autophagy. Nanomater. 2021;11:2. doi:10.3390/nano11020384

93. Xia QH, Zheng LP, Zhao PF, Wang JW. Biosynthesis of silver nanoparticles using Artemisia annua callus for inhibiting stem-end bacteria in cut carnation flowers. IET Nanobiotechnology. 2017;11 (2):185-192. doi:10.1049/iet-nbt.2015.0125

94. Roy P, Das B, Mohanty A, Mohapatra S. Green synthesis of silver nanoparticles using Azadirachta indica leaf extract and its antimicrobial study. Appl Nanosci. 2017;7(8):843-850. doi:10.1007/ s13204-017-0621-8

95. Sharma JK, Srivastava P, Ameen S, Akhtar MS, Sengupta SK, Singh G. Phytoconstituents assisted green synthesis of cerium oxide nanoparticles for thermal decomposition and dye remediation. Mater Res Bull. 2017;91:98-107. doi:10.1016/J. MATERRESBULL.2017.03.034

96. Nagar N, Devra V. Green synthesis and characterization of copper nanoparticles using Azadirachta indica leaves. Mater Chem Phys. 2018;213:44-51. doi:10.1016/j.matchemphys.2018.04.007

97. Dulta K, Koşarsoy Ağçeli G, Chauhan P, Jasrotia R, Chauhan PK. A novel approach of synthesis zinc oxide nanoparticles by bergenia ciliata rhizome extract: antibacterial and anticancer potential. J Inorg Organomet Polym Mater. 2021;31 (1):180-190. doi:10.1007/s10904-020-01684-6

98. Ayodhya D, Veerabhadram G. One-pot green synthesis, characterization, photocatalytic, sensing and antimicrobial studies of Calotropis gigantea leaf extract capped CdS NPs. Mater Sci Eng B. 2017;225:33-44. doi:10.1016/j.mseb.2017.08.008

99. Singh D, Kumar V, Yadav E, et al. One-pot green synthesis and structural characterisation of silver nanoparticles using aqueous leaves extract of Carissa carandas: antioxidant, anticancer and antibacterial activities. IET Nanobiotechnology. 2018;12 (6):748-756

100. Osibe DA, Chiejina NV, Ogawa K, Aoyagi H. Stable antibacterial silver nanoparticles produced with seed-derived callus extract of Catharanthus roseus. Artif Cells Nanomed Biotechnol. 2018;46 (6):1266-1273. doi:10.1080/21691401.2017.1367927

101. Gupta M, Tomar RS, Kaushik S, Mishra RK, Sharma D. Effective antimicrobial activity of green $\mathrm{ZnO}$ nano particles of Catharanthus roseus. Front Microbiol. 2018;9:2030. doi:10.3389/fmicb.2018.02030
102. Azizi S, Mohamad R, Mahdavi Shahri M. Green microwave-assisted combustion synthesis of zinc oxide nanoparticles with Citrullus colocynthis (L.) Schrad: characterization and biomedical applications. Molecules. 2017;22(2):301. doi:10.3390/ molecules 22020301

103. John Sushma N, Prathyusha D, Swathi G, et al. Facile approach to synthesize magnesium oxide nanoparticles by using Clitoria ternatea - characterization and in vitro antioxidant studies. Appl Nanosci. 2016;6(3):437-444. doi:10.1007/s13204-015-0455-1

104. Fatimah I, Sahroni I, Muraza O, Doong R. One-pot biosynthesis of $\mathrm{SnO} 2$ quantum dots mediated by Clitoria ternatea flower extract for photocatalytic degradation of rhodamine B. $J$ Environ Chem Eng. 2020;8(4):103879. doi:10.1016/j.jece.2020.103879

105. Vilas V, Philip D, Mathew J. Essential oil mediated synthesis of silver nanocrystals for environmental, anti-microbial and antioxidant applications. Mater Sci Eng C. 2016;61:429-436. doi:10.1016/J.MSEC.2015.12.083

106. Bathula C, Kumar KA, et al. Ultrasonically driven green synthesis of palladium nanoparticles by Coleus amboinicus for catalytic reduction and Suzuki-Miyaura reaction. Colloids Surfaces B Biointerfaces. 2020;192:111026. doi:10.1016/j.colsurfb.2020.111026

107. Khan FU, Chen Y, Khan NU, et al. Visible light inactivation of E. coli, Cytotoxicity and ROS determination of biochemically capped gold nanoparticles. Microb Pathog. 2017;107:419-424. doi:10.1016/J.MICPATH.2017.04.024

108. Vijayan R, Joseph S, Mathew B. Costus speciosus rhizome extract mediated synthesis of silver and gold nanoparticles and their biological and catalytic properties. Inorg Nano-Metal Chem. 2019;49(8):249-259. doi:10.1080/24701556.2019.1661439

109. Bagherzade G, Tavakoli MM, Namaei MH. Green synthesis of silver nanoparticles using aqueous extract of saffron (Crocus sativus L.) wastages and its antibacterial activity against six bacteria. Asian Pac J Trop Biomed. 2017;7(3):227-233. doi:10.1016/j.apjtb.2016.12.014

110. Aboyewa JA, Sibuyi NRS, Meyer M, Oguntibeju OO. Gold nanoparticles synthesized using extracts of cyclopia intermedia, commonly known as honeybush, amplify the cytotoxic effects of doxorubicin. Nanomater. 2021;11:1. doi:10.3390/nano11010132

111. Ruíz-Baltazar Á de J, Reyes-López SY, Mondragón-Sánchez M de L, Estevez M, Hernández-Martinez AR, Pérez R. Biosynthesis of Ag nanoparticles using Cynara cardunculus leaf extract: evaluation of their antibacterial and electrochemical activity. Results Phys. 2018;11:1142-1149. doi:10.1016/j.rinp.2018.11.032

112. Suresh S, Ilakiya R, Kalaiyan G, et al. Green synthesis of copper oxide nanostructures using Cynodon dactylon and Cyperus rotundus grass extracts for antibacterial applications. Ceram Int. 2020;46(8):12525-12537. doi:10.1016/j.ceramint.2020.02.015

113. Anandan M, Poorani G, Boomi P, et al. Green synthesis of anisotropic silver nanoparticles from the aqueous leaf extract of Dodonaea viscosa with their antibacterial and anticancer activities. Process Biochem. 2019;80:80-88. doi:10.1016/j. procbio.2019.02.014

114. Jabir MS, Hussien AA, Sulaiman GM, et al. Green synthesis of silver nanoparticles from Eriobotrya japonica extract: a promising approach against cancer cells proliferation, inflammation, allergic disorders and phagocytosis induction. Artif Cells Nanomed Biotechnol. 2021;49 (1):48-60. doi:10.1080/21691401.2020.1867152

115. Francis S, Joseph S, Koshy EP, Mathew B. Microwave assisted green synthesis of silver nanoparticles using leaf extract of elephantopus scaber and its environmental and biological applications. Artif Cells Nanomed Biotechnol. 2018;46 (4):795-804. doi:10.1080/21691401.2017.1345921

116. Abbai R, Mathiyalagan R, Markus J, et al. Green synthesis of multifunctional silver and gold nanoparticles from the oriental herbal adaptogen: Siberian ginseng. Int $J$ Nanomedicine. 2016;11:3131-3143. doi:10.2147/IJN.S108549 
117. Balaji S, Guda R, Mandal BK, Kasula M, Ubba E, Khan F-RN. Green synthesis of nano-titania ( $\mathrm{TiO} 2 \mathrm{NPs}$ ) utilizing aqueous Eucalyptus globulus leaf extract: applications in the synthesis of 4H-pyran derivatives. Res Chem Intermed. 2019. doi:10.1007/ s11164-018-03720-0

118. Balamurugan M, Saravanan S. Green synthesis of silver nanoparticles by using eucalyptus globulus leaf extract. $J$ Inst Eng Ser A. 2017;98(4):461-467. doi:10.1007/s40030-017-0236-9

119. Ahmad H, Venugopal K, Rajagopal K, et al. Green synthesis and characterization of zinc oxide nanoparticles using eucalyptus globules and their fungicidal ability against pathogenic fungi of apple orchards. Biomol. 2020;10:3. doi:10.3390/biom10030425

120. Aksu Demirezen D, Yıldız YŞ, Yılmaz Ş, Demirezen yılmaz D. Green synthesis and characterization of iron oxide nanoparticles using Ficus carica (common fig) dried fruit extract. $J$ Biosci Bioeng. 2019;127(2):241-245. doi:10.1016/j.jbiosc.2018.07.024

121. Gopinath K, Kumaraguru S, Bhakyaraj K, et al. Green synthesis of silver, gold and silver/gold bimetallic nanoparticles using the Gloriosa superba leaf extract and their antibacterial and antibiofilm activities. Microb Pathog. 2016;101:1-11. doi:10.1016/J. MICPATH.2016.10.011

122. Naika HR, Lingaraju K, Manjunath K, et al. Green synthesis of $\mathrm{CuO}$ nanoparticles using Gloriosa superba $\mathrm{L}$. extract and their antibacterial activity. J Taibah Univ Sci. 2015;9(1):7-12. doi:10.1016/j.jtusci.2014.04.006

123. Rokade SS, Joshi KA, Mahajan K, et al. Gloriosa superba mediated synthesis of platinum and palladium nanoparticles for induction of apoptosis in breast cancer. Bioinorg Chem Appl. 2018;2018:4924186. doi:10.1155/2018/4924186

124. Talebpour F, Ghahghaei A. Effect of green synthesis of gold nanoparticles (AuNPs) from Hibiscus sabdariffa on the aggregation of $\alpha$-lactalbumin. Int J Pept Res Ther. 2020. doi:10.1007/ s10989-020-10023-9

125. Kumar V, Singh S, Srivastava B, Bhadouria R, Singh R. Green synthesis of silver nanoparticles using leaf extract of Holoptelea integrifolia and preliminary investigation of its antioxidant, anti-inflammatory, antidiabetic and antibacterial activities. $J$ Environ Chem Eng. 2019;7(3):103094. doi:10.1016/j. jece.2019.103094

126. Botcha S, Prattipati SD. Callus extract mediated green synthesis of silver nanoparticles, their characterization and cytotoxicity evaluation against MDA-MB-231 and PC-3 cells. Bionanoscience. 2020;10(1):11-22. doi:10.1007/s12668-01900683-3

127. Aritonang HF, Koleangan H, Wuntu AD. Synthesis of silver nanoparticles using aqueous extract of medicinal plants' (Impatiens balsamina and Lantana camara) fresh leaves and analysis of antimicrobial activity. Callaway TR, ed. Int J Microbiol. 2019;2019:8642303. doi:10.1155/2019/8642303

128. Khaled JM, Alharbi NS, Kadaikunnan S, et al. Green synthesis of $\mathrm{Ag}$ nanoparticles with anti-bacterial activity using the leaf extract of an African medicinal plant, Ipomoea asarifolia (Convolvulaceae). $\quad J \quad$ Clust $\quad$ Sci. 2017;28(5):3009-3019. doi:10.1007/s10876-017-1271-4

129. Pachaiappan R, Rajendran S, Ramalingam G, Vo D-VN, Priya PM, Soto-Moscoso M. Green synthesis of zinc oxide nanoparticles by Justicia adhatoda leaves and their antimicrobial activity. Chem Eng Technol. 2021;44(3):551-558. doi:10.1002/ ceat. 202000470

130. Patil SP, Kumbhar ST. Antioxidant, antibacterial and cytotoxic potential of silver nanoparticles synthesized using terpenes rich extract of Lantana camara L. leaves. Biochem Biophys Reports. 2017;10:76-81. doi:10.1016/j.bbrep.2017.03.002
131. Vijayakumar S, Vaseeharan B, Malaikozhundan B, Shobiya M. Laurus nobilis leaf extract mediated green synthesis of $\mathrm{ZnO}$ nanoparticles: characterization and biomedical applications. Biomed Pharmacother. 2016;84:1213-1222. doi:10.1016/J. BIOPHA.2016.10.038

132. Mahmoudi R, Aghaei S, Salehpour Z, et al. Antibacterial and antioxidant properties of phyto-synthesized silver nanoparticles using Lavandula stoechas extract. Appl Organomet Chem. 2020;34(2):e5394. doi:10.1002/aoc.5394

133. Dadashpour M, Firouzi-Amandi A, Pourhassan-Moghaddam M, et al. Biomimetic synthesis of silver nanoparticles using Matricaria chamomilla extract and their potential anticancer activity against human lung cancer cells. Mater Sci Eng C. 2018;92:902-912. doi:10.1016/j.msec.2018.07.053

134. Dogru E, Demirbas A, Altinsoy B, Duman F, Ocsoy I. Formation of Matricaria chamomilla extract-incorporated Ag nanoparticles and size-dependent enhanced antimicrobial property. J Photochem Photobiol B Biol. 2017;174:78-83. doi:10.1016/j. jphotobiol.2017.07.024

135. Duman F, Ocsoy I, Kup FO. Chamomile flower extract-directed $\mathrm{CuO}$ nanoparticle formation for its antioxidant and DNA cleavage properties. Mater Sci Eng C. 2016;60:333-338. doi:10.1016/j. msec.2015.11.052

136. Bouafia A, Laouini SE. Green synthesis of iron oxide nanoparticles by aqueous leaves extract of Mentha Pulegium L.: effect of ferric chloride concentration on the type of product. Mater Lett. 2020;265:127364. doi:10.1016/j.matlet.2020.127364

137. Morales-Lozoya V, Espinoza-Gómez H, Flores-LópezL Z, et al. Study of the effect of the different parts of Morinda citrifolia L. (noni) on the green synthesis of silver nanoparticles and their antibacterial activity. Appl Surf Sci. 2021;537:147855. doi:10.1016/j.apsusc.2020.147855

138. Surendra TV, Roopan SM, Arasu MV, Al-Dhabi NA, Rayalu GM. RSM optimized Moringa oleifera peel extract for green synthesis of M. oleifera capped palladium nanoparticles with antibacterial and hemolytic property. $J$ Photochem Photobiol B Biol. 2016;162:550-557. doi:10.1016/J. JPHOTOBIOL.2016.07.032

139. Bindhu MR, Umadevi M, Esmail GA, Al-Dhabi NA, Arasu MV. Green synthesis and characterization of silver nanoparticles from Moringa oleifera flower and assessment of antimicrobial and sensing properties. $J$ Photochem Photobiol B Biol. 2020;205:111836. doi:10.1016/j.jphotobiol.2020.111836

140. Katata-Seru L, Moremedi T, Aremu OS, Bahadur I. Green synthesis of iron nanoparticles using Moringa oleifera extracts and their applications: removal of nitrate from water and antibacterial activity against Escherichia coli. J Mol Liq. 2018;256:296-304. doi:10.1016/j.molliq.2017.11.093

141. Das PE, Abu-Yousef IA, Majdalawieh AF, Narasimhan S, Poltronieri P. Green synthesis of encapsulated copper nanoparticles using a hydroalcoholic extract of Moringa oleifera leaves and assessment of their antioxidant and antimicrobial activities. Molecules. 2020;25(3):555. doi:10.3390/molecules25030555

142. Das PE, Majdalawieh AF, Abu-Yousef IA, Narasimhan S, Poltronieri P. Use of A hydroalcoholic extract of Moringa oleifera leaves for the green synthesis of bismuth nanoparticles and evaluation of their anti-microbial and antioxidant activities. Mater. 2020;13(4):876. doi:10.3390/ma13040876

143. Some S, Sarkar B, Biswas K, et al. Bio-molecule functionalized rapid one-pot green synthesis of silver nanoparticles and their efficacy toward the multidrug resistant (MDR) gut bacteria of silkworms (Bombyx mori). RSC Adv. 2020;10 (38):22742-22757. doi:10.1039/D0RA03451G 
144. Vijayan R, Joseph S, Mathew B. Green synthesis of silver nanoparticles using Nervalia zeylanica leaf extract and evaluation of their antioxidant, catalytic, and antimicrobial potentials. Part Sci Technol. 2019;37(7):809-819. doi:10.1080/ 02726351.2018 .1450312

145. Francis S, Joseph S, Koshy EP, Mathew B. Green synthesis and characterization of gold and silver nanoparticles using Mussaenda glabrata leaf extract and their environmental applications to dye degradation. Environ Sci Pollut Res. 2017;24(21):17347-17357. doi:10.1007/s11356-017-9329-2

146. Francis S, Joseph S, Koshy EP, Mathew B. Synthesis and characterization of multifunctional gold and silver nanoparticles using leaf extract of Naregamia alata and their applications in the catalysis and control of mastitis. New J Chem. 2017;41 (23):14288-14298. doi:10.1039/C7NJ02453C

147. Brahmachari G, Sarkar S, Ghosh R, et al. Sunlight-induced rapid and efficient biogenic synthesis of silver nanoparticles using aqueous leaf extract of Ocimum sanctum Linn. with enhanced antibacterial activity. Org Med Chem Lett. 2014;4(1):18. doi:10.1186/s13588-014-0018-6

148. Pandian CJ, Palanivel R, Dhananasekaran S. Green synthesis of nickel nanoparticles using Ocimum sanctum and their application in dye and pollutant adsorption. Chinese J Chem Eng. 2015;23 (8):1307-1315. doi:10.1016/j.cjche.2015.05.012

149. De Matteis V, Rizzello L, Ingrosso C, et al. Cultivar-dependent anticancer and antibacterial properties of silver nanoparticles synthesized using leaves of different Olea Europaea trees. Nanomater. 2019;9(11):1544. doi:10.3390/nano9111544

150. Şeker Karatoprak G, Aydin G, Altinsoy B, Altinkaynak C, Koşar M, Ocsoy I. The effect of Pelargonium endlicherianum Fenzl. root extracts on formation of nanoparticles and their antimicrobial activities. Enzyme Microb Technol. 2017;97:21-26. doi:10.1016/j.enzmictec.2016.10.019

151. Carson L, Bandara S, Joseph M, et al. Green synthesis of silver nanoparticles with antimicrobial properties using Phyla dulcis plant extract. Foodborne Pathog Dis. 2020. doi:10.1089/ fpd.2019.2714

152. Dinesh M, Roopan SM, Selvaraj CI, Arunachalam P. Phyllanthus emblica seed extract mediated synthesis of PdNPs against antibacterial, heamolytic and cytotoxic studies. $J$ Photochem Photobiol B Biol. 2017;167:64-71. doi:10.1016/J. JPHOTOBIOL.2016.12.012

153. Masum MMI, Siddiqa MM, Ali KA, et al. Biogenic synthesis of silver nanoparticles using Phyllanthus emblica fruit extract and its inhibitory action against the pathogen Acidovorax oryzae strain RS-2 of rice bacterial brown stripe. Front Microbiol. 2019;10:820. doi:10.3389/fmicb.2019.00820

154. Shanavas S, Priyadharsan A, Karthikeyan S, et al. Green synthesis of titanium dioxide nanoparticles using Phyllanthus niruri leaf extract and study on its structural, optical and morphological properties. Mater Today Proc. 2019. doi:10.1016/j. matpr.2019.06.715

155. Alsalhi MS, Devanesan S, Alfuraydi AA, et al. Green synthesis of silver nanoparticles using Pimpinella anisum seeds: antimicrobial activity and cytotoxicity on human neonatal skin stromal cells and colon cancer cells. Int J Nanomedicine. 2016;11:4439-4449. doi:10.2147/IJN.S113193
156. Shobana C, Rangasamy B, Poopal RK, Renuka S, Ramesh M. Green synthesis of silver nanoparticles using Piper nigrum: tissue-specific bioaccumulation, histopathology, and oxidative stress responses in Indian major carp Labeo rohita. Environ Sci Pollut Res. 2018;25(12):11812-11832. doi:10.1007/s11356-0181454-z

157. Khatua A, Priyadarshini E, Rajamani P, et al. Phytosynthesis, characterization and fungicidal potential of emerging gold nanoparticles using Pongamia pinnata leave extract: a novel approach in nanoparticle synthesis. J Clust Sci. 2020;31(1):125-131. doi:10.1007/s10876-019-01624-6

158. Ghramh HA, Khan KA, Ibrahim EH, Setzer WN. Synthesis of gold nanoparticles (AuNPs) using Ricinus communis leaf ethanol extract, their characterization, and biological applications. Nanomater. 2019;9(5):765. doi:10.3390/nano9050765

159. Jafarirad S, Mehrabi M, Divband B, Kosari-Nasab M. Biofabrication of zinc oxide nanoparticles using fruit extract of Rosa canina and their toxic potential against bacteria: a mechanistic approach. Mater Sci Eng C. 2016;59:296-302. doi:10.1016/J.MSEC.2015.09.089

160. Perugu S, Nagati V, Bhanoori M. Green synthesis of silver nanoparticles using leaf extract of medicinally potent plant Saraca indica: a novel study. Appl Nanosci. 2016;6(5):747-753. doi:10.1007/s13204-015-0486-7

161. Shaik M, Albalawi G, Khan S, et al. "Miswak" based green synthesis of silver nanoparticles: evaluation and comparison of their microbicidal activities with the chemical synthesis. Molecules. 2016;21(11):1478. doi:10.3390/molecules21111478

162. Miri A, Dorani N, Darroudi M, Sarani M. Green synthesis of silver nanoparticles using Salvadora persica L. and its antibacterial activity. Cell Mol Biol (Noisy-Le-Grand). 2016;62(9):46-50.

163. Miri A, Sarani M. Biosynthesis and cytotoxic study of synthesized zinc oxide nanoparticles using Salvadora persica. Bionanoscience. 2019;9(1):164-171. doi:10.1007/s12668-018-0579-3

164. Francis S, Koshy EP, Mathew B. Green synthesis of Stereospermum suaveolens capped silver and gold nanoparticles and assessment of their innate antioxidant, antimicrobial and antiproliferative activities. Bioprocess Biosyst Eng. 2018;41 (7):939-951. doi:10.1007/s00449-018-1925-0

165. Ajitha B, Reddy YAK, Lee Y, Kim MJ, Ahn CW. Biomimetic synthesis of silver nanoparticles using Syzygium aromaticum (clove) extract: catalytic and antimicrobial effects. Appl Organomet Chem. 2019;33(5):e4867. doi:10.1002/aoc.4867

166. Andra S, Balu S, Ramoorthy R, Muthalagu M, Manisha VS. Terminalia bellerica fruit extract mediated synthesis of silver nanoparticles and their antimicrobial activity. Mater Today Proc. 2019;9:639-644. doi:10.1016/j.matpr.2018.10.387

167. Selvam K, Sudhakar C, Govarthanan M, et al. Eco-friendly biosynthesis and characterization of silver nanoparticles using Tinospora cordifolia (Thunb.) Miers and evaluate its antibacterial, antioxidant potential. J Radiat Res Appl Sci. 2017;10(1):6-12. doi:10.1016/j.jrras.2016.02.005

168. Tripathi D, Modi A, Narayan G, Rai SP. Green and cost effective synthesis of silver nanoparticles from endangered medicinal plant Withania coagulans and their potential biomedical properties. Mater Sci Eng C. 2019;100:152-164. doi:10.1016/j. msec.2019.02.113 
169. Alagesan V, Venugopal S. Green Synthesis of selenium nanoparticle using leaves extract of Withania somnifera and its biological applications and photocatalytic activities. Bionanoscience. 2019;9 (1):105-116. doi:10.1007/s12668-018-0566-8

170. Vijaya JJ, Jayaprakash N, Kombaiah K, et al. Bioreduction potentials of dried root of Zingiber officinale for a simple green synthesis of silver nanoparticles: antibacterial studies. $J$ Photochem Photobiol B Biol. 2017;177:62-68. doi:10.1016/j. jphotobiol.2017.10.007
171. Aljabali AAA, Akkam Y, Al Zoubi MS, et al. Synthesis of gold nanoparticles using leaf extract of Ziziphus zizyphus and their antimicrobial activity. Nanomater. 2018;8:3. doi:10.3390/ nano8030174

172. Kumar VS, Navaratnam V, Rajasekaran A, et al. Isolation and characterization of glucosamine from Azadirachta indica leaves: an evaluation of immunostimulant activity in mice. Asian Pac J Trop Biomed. 2012;2(3):S1561-S1567. doi:10.1016/S2221-1691(12)60453-5

\section{Publish your work in this journal}

Nanotechnology, Science and Applications is an international, peerreviewed, open access journal that focuses on the science of nanotechnology in a wide range of industrial and academic applications. It is characterized by the rapid reporting across all sectors, including engineering, optics, bio-medicine, cosmetics, textiles, resource sustainability and science. Applied research into nano-materials, particles, nano-structures and fabrication, diagnostics and analytics, drug delivery and toxicology constitute the primary direction of the journal. The manuscript management system is completely online and includes a very quick and fair peer-review system, which is all easy to use. Visit http://www.dovepress.com/testimonials.php to read real quotes from published authors. 